\title{
LES HELMINTHES PARASITES DE COLÉOPTÈRES CHRYSOMÉLIDES
}

\section{Par Pierre JOLIVET et Jean THÉOdORIDĖS}

On connait relativement peu de chose des Helminthes parasites de Coléoptères et tout particulièrement sur ceux de Chrysomélides. En dehors des listes de Van Zwaluwenburg (1928) et de La Rivers (1949) (1), forcément incomplètes, rien n'a été publié sur cette question (2) et nous avons donc pensé que cette note serait de quelque utilité pour les biologistes qui s'intéressent aux relations entre Helminthes et Insectes.

Vu le petit nombre d'hôtes connus, les parasites seront énumérés d'après l'ordre systématique de ceux-ci :

\section{CHRYSOMELIDE s. str.}

1. Gastroidea cyanea Melsh. - Denton (1945) signale un cas très curieux où les métacercaires d'un Trématode peuvent vivre chez un Coléoptère terrestre (fait très rare et dont ce serait le seul exemple) ; il s'agit de Brachylecithum americanum Denton (Dicrocœliidæ) (3), dont une à six métacercaires non enkystées ont été rencontrées dans la cavité abdominale des larves de ce Coléoptère.

Cette infestation avait été réalisée expérimentalement en exposant les larves et adultes de G. cyanea à des cercaires répandues sur les Rumex leur servant de nourriture ; l'auteur ne peut préciser si ces dernières pénétraient par voie orale ou d'une autre manière. Quant aux adultes soumis aux mêmes conditions que les larves, ils ne contenaient aucune métacercaire.

(1) Nous tenons à remercier ici MM. Van Zwaluwenburg et La Rivers (Honolulu, Hawaï et Reno, Nevada) qui nous ont si aimablement communiqué leurs publications, difficiles à se procurer en France.

(2) L'un de nous (Jolivet, 1946, 1947) a cependant cité quelques-uns des Helminthes signalés d'espèces se rencontrant en France.

(3) Cette espèce à l'état adulte parasite les canaux biliaires de divers Passereaux : Cassidis mexicanus, prosopidicola, Quiscalus versicolor, Sturnella miagna, Corvus brachyrhynchos, Cyanocitta cristata.

Annales de Parasitologie, T. XXV, $\mathrm{N}^{\circ} 4 .-1950$. 
Il semblerait donc que $G$. cyanea puisse servir de second hôte intermédiaire de ce Trématode, et que les oiseaux s'infestent en mangeant les Coléoptères.

Diabrotica duodecimpunctata (Fab.) peut jouer le même rôle, comme nous le verrons plus loin.

Les autres Helminthes mentionnés ici ont des Cḥrysomélides comme hôte primaire et unique, et, dans le cas des Mermithidés et des Gordiacés, ce sont leurs larves qui sont parasites.

2. Timarcha tenebricosa F. - Parachordodes violaceus (Baird) est signalé de cet hôte en France (Dorier, 1930). Ce Gordiacé parasite également d'autres Insectes.

3. Leptinotarsa decemlineata Say. - Abrahams (1938) signale des Mermithidés rapportés provisoirement au genre Hexamermis parasitant tous les stades du Doryphore près de Baden (Allemagne). Chez les Insectes infestés, se produit une réduction des gonades, mais il ne semble pas que celle-ci soit suffisante pour faire périr l'hôte, car autrement ce parasite aurait été utilisé dans la lutte biologique contre ce redoutable fléau des cultures.

4. Chrysochloa collucens Dan. - Un Mermithidé indéterminé (peut-être un Hexamermis) parasite ce Coléoptère en Normandie (J. Bourdon in litt.).

5. Chrysomela populi L. (= Melasoma ou Lina populi L.) (1). Rauther (1907), dans un important travail sur l'anatomie interne de Mermis albicans V. Sieb. (2), mentionne ce Chrysomélide comme hôte de ce Nématode.

Les Coléoptères avaient été récoltés pendant l'été 1903, au bord d'un ruisseau à Tübingen (Allemagne), et étaient très parasités (jusqu'à trois Helminthes par individu), les larves se montrant plus atteintes que les adultes et les Mermis s'en échappant par les stigmates.

On avait donc trouvé des Mermithidés chez cette espèce avant Devantoy (1948 $a$ et $b)$, qui dit $(1948 b):$ :Aucun Nématode n'avait

(1) Nous adoptons ici, à la suite de la plupart des spécialistes étrangers, la terminologie proposée par Maulik en 1925, à savoir : Chrysomela L. pour l'ancien genre Melasoma Steph., Chrysolina Motsch. devant remplacer Chrysomela auct. nec L.

(2) Ce Mermis a été mis ensuite dans le genre Hexamermis créé par Steiner en 1924 et caractérisé par la présence de six bourrelets longitudinaux (longitudinal chords) et est figuré comme tel par Filipjev et Schuurmans Stekhoven (1941), d'après Hagmeier (1912), qui a illustré les détails d'un mâle de cette espèce mais ne mentionne aucun Coléoptère parmi les hôtes des larves. 
été observé chez Melasoma populi »; cet auteur signale un Hexamermis sp. déterminé par R.-Ph. Dollfus, qui le mentionne à propos d'une note sur un Allomermis de fourmi (Dollfus, 1945).

Les récoltes de Devantoy ont été faites en juin-juillet 1944 et 1945 et proviennent de larves récoltées au lieu dit "les Grands Bois 》, à $7 \mathrm{~km}$. au S.-E. de Belfort (Territoire de Belfort), les Helminthes quittant leur hôte mort ou mourant pour vivre en liberté. Ces Hexamermis ont pu être déterminés génériquement du fait qu'un des

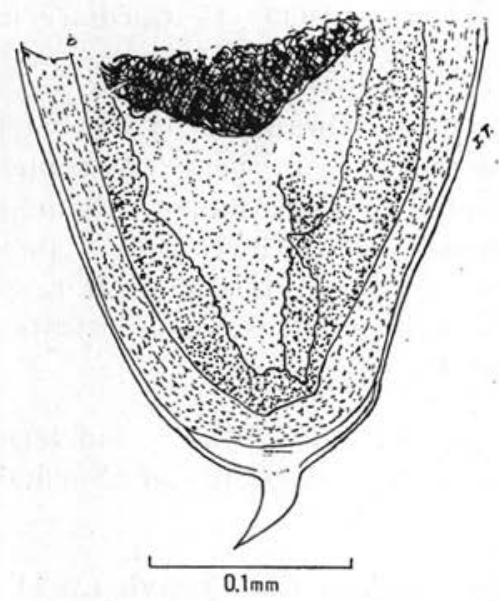

Fig. 1. - Extrémité postérieure d'une larve d'Hexamermis sp. provenant de Chrysomela populi L. (Gualba, Espagne), montrant la corne caudale caractéristique de ce genre. (Original).

exemplaires était à l'état larvaire et présentait la corne caudale caractéristique des larves de ce genre, comme nous allons le voir lout de suite.

Enfin, M. E. Balcells (Université de Barcelone, Espagne) nous a aimablement communiqué (et nous l'en remercions ici) un Mermithidé d'environ $6,5 \mathrm{~cm}$. de long et provenant d'une larve de cet hôte recueillie avec d'autres individus de cette espèce à Gualba (altititude $300 \mathrm{~m}$.), non loin de la Sierra de Montseny (Province de Barcelone), pendant l'été 1948.

Cet exemplaire étudié par l'un de nous (Théodoridès) est en parfait état et présente à l'extrémité postérieure l'appendice en forme de corne caractéristique du genre Hexamermis (1) (fig. 1). Peut-être

(1) La diagnose des larves de ce genre est donnée par Steiner in Dollfus, op. cit.) : « On reconnaît les larves de ce genre à un court appendice en forme de corne à l'extrémité postérieure. 
s'agit-il ici du même Hexamermis que celui trouvé en Allemagne et en France, qui, dans le premier cas, serait $H$. albicans V. Sieb. et compterait $C$. populi parmi ses hôtes réguliers (1).

\section{GALERUCIDAE}

6. Agelastica alni L. - Trois Mermithidés ont été signalés chez cette Galéruque, mais, en l'absence de types, il ne nous a pas été permis de débrouiller complètement la synonymie vraisemblable :

Filaria chrysomela Holten.

Hôte cité par Holten (1797) et Rudolphi (1809).

Mermis galleruca-alni Dies.

Hôte cité par Diesing (1861) et Von Linstow (1878).

Mermis nigrescens Duj. 1842.

Cité par Von Linstow (1898) comme hôte douteux.

L'un de nous ayant pu consulter le Systema helminthum de Diesing (1861), y a lu ce qui suit :

$a$. Ce Mermithidé y est en fait cité comme Gordius gallerucæ alni Dies. et non Mermis g.a. comme le transcrit Van Zwaluwenburg (1928), bien qu'il s'agisse ici sûrement d'un Mermithidé.

b. Il est bien synonyme de Filaria chrysomela Holten.

c. Holten avait trouvé cet Helminthe dans la cavité abdominale du Coléoptère, au Danemark.

Enfin, M. Balcells nous a communiqué deux exemplaires de Mermithidés recueillis de cet hôte dans la même localité (Gualba) et à la même époque que l'Hexamermis sp. de Chrysomela populi menlionné ci-dessus.

Ces deux Helminthes mesurent respectivement $5,8 \mathrm{~cm}$. et $4,5 \mathrm{~cm}$., mais sont malheureusement endommagés, ayant été attaqués après leur mort (2) par un Aspergillus sp. (A. Bulliard det.). Cependant, chez l'un d'eux, la présence indubitable d'un appendice en forme de corne laisse penser qu'il pourrait s'agir d'un Hexamermis sp.

(1) Les Mermithides de Chrysomela L. doivent être assez rares : C. tremula F. était très abondant dans une coupe de peupliers à Poigny-la-Forêt (S.-et-0.) et 65 exemplaires examinés par l'un de nous (J.T.) le 27-6-1948 ne contenaient aucun Helminthe.

(2) Ces 2 exemplaires ainsi que celui de C. populi ont été trouvés au laboratoire peu de temps après la récolte de ces Chrysomélides sur le terrain. 
6 bis. Galeruca (= Adimonia) tanaceti L. - Diesing (1861) mentionne un Gordius gallerucæ tanaceti Dies. décrit auparavant comme Filaria chrysomelæ tanaceti Fröl. et qui doit être en fait un Mermithidé. Cet Helminthe aurait été trouvé dans la cavité abdominale de l'hôte en Allemagne (Frölich) et en Angleterre (Hope). Cet hôte n'a jamais été mentionné depuis et n'est pas cité par Van Zwaluwenburg (1928) ; nous le donnons done ici sous réserve et le mettons en appendice au Chrysomélide précédent, à la suite duquel il figure dans l'ouvrage de Diesing.

7. Diabrotica duodecimpunctata (Fab.), 8. Diabrotica trivittata (Mann.), 9. Diabrotica vittata (Fab.). - Ces trois espèces vivent à l'est des Etats-Unis et sont parasitées par un Nématode, Anguillulidx: Howardula benigna Cobb 1921.

Ce dernier se rencontre dans l'abdomen, le thorax et même la tête des hôtes adultes; l'infestation peut atteindre 70 p. 100 et la moyenne est de $20 \mathrm{p}$. 100 . Les Coléoptères parasités sont plus petits et moins vigoureux, et les femelles infestées moins fertiles.

Un de ces Chrysomélides peut héberger 250 Nématodes adultes et 13.000 larves furent obtenues d'un seul individu (Cobb, 1921, 1922). Ces dernières quittent l'hôte avec les œufs (de 6 à 50 par œuf) et, lorsque la larve du Coléoptère éclôt, elles pénètrent à l'intérieur de celle-ci.

Ce Nématode est un facteur de contrôle de grande importance économique dans la lutte contre les Diabrotica, fléaux des cultures. Denton (1945) a obtenu expérimentalement des métacercaires du 'Trématode Brachylecithum americanum, déjà mentionné plus haut, chez un $D$. duodecimpunctata, qui avait été exposé pendant deux jours en présence d'un Gastéropode infesté de cercaires.

Trois des métacercaires étaient incluses dans des kystes hyalins, tandis que les autres étaient libres dans les corps gras. Peut-être même qu'à l'infestation expérimentale s'en était jointe une naturelle.

Quoi qu'il en soit, D. duodecimpunctata peut être le deuxième hôte intermédiaire de ce Trématode au même titre que Gastroidea cyanea.

\section{HALTICIDE}

10. Phyllotreta undulata Kutsch. - Howardula phyllotretæ Oldh. est décrit de cette Haltise dans le Hertfordshire (Angleterre) (Oldham, 1933) et 70 p. 100 des Coléoptères étudiés furent trouvés 
infestés. Les dernierś stades larvaires de ce Nématode pénètrent dans les ovaires et les oviductes des hôtes apparemment pour être déposés avec leurs œufs.

Les genitalia des Chrysomélides mâles ne sont pas parasités et la façon dont l'Helminthe s'échappe de ces derniers n'est pas connue. Les Insectes infestés montrent une réduction générale dans leur développement et leur fécondité.

Des exemplaires d'Allemagne (Schleswig-Holstein) furent aussi trouvés infestés. On remarquera l'analogie entre la biologie de ce Nématode et celle de $H$. benigna Cobb, parasite des Diabrotica américaines. Filipjev et Schuurmans Stekhoven (1941) signalent ce même Nématode à Peterhof (environs de Léningrad, U.R.S.S.), où 50 p. 100 des Coléoptères étaient infestés.

De même, Pjatakova (1928) et Dournovo (1927) ont trouvé cette espèce respectivement à Mléew et Moscou. Cette dernière possède donc apparemment une vaste aire de répartition.

Oldham (1933) a trouvé, en plus des Howardula, un Mermithidé indéterminé chez un ơ de Phyllotreta undulata; c'était une femelle immature, donc impossible à déterminer.

11. Phyllotreta nigripes F., 12. Phyllotreta atra F., 13. Phyllotreta cruciferæ Gœze, 14. Phyllotreta vittula Redt. - Toutes ces espèces sont parasitées par Howardula phyllotretæ.

En ce qui concerne $P$. atra, Newton (1931) a signalé un Mermis sp. chez une $q$ adulte et un petit Nématode, apparemment $H$. phyllotretæ, qui se trouve en grand nombre dans la cavité abdominale des larves, nymphes et adultes. Les recherches de Newton ont eu lieu en 1927 à Wye (Kent).

Seule Phyllotreta nemorum L. ne se montre pas infestée par H. phyllotretæ dans ses stades larvaires et à l'état adulte (Oldham, 1935) (1) ; ce dernier auteur note également la rareté de la coexistence d'Hyménoptères parasites et de Nématodes dans le même hôte.

15. Phyllotreta pusilla Horn. - Chittenden et Marsh (1920) ont signalé des Nématodes parasitant cette espèce. D'après le nombre de parasites par Coléoptère (200 à 500), il s'agirait d'un Anguillulide, peut-être un Howardula.

16. Phyllotreta sp. - Pjatakova (1928) a trouvé, en plus des Howardula, chez une Phyllotreta, un Nématode de plusieurs centi-

(1) Dans sa note de 1935 , Oldham donne les pourcentages suivants d'individus parasités : $P$. atra 7,5 p. $100 ; P$. cruciferæ 3,58 p. $100 ; P$. nigripes 11,19 p. 100 ; P. undulata 42,40 p. 100 ; P. vittula 50 p. 100.

annales de Parasitologie, $\mathrm{T} . \mathrm{XXV}, \mathrm{N}^{\circ} 4 .-1950$. 
mètres qui, d'après la figure qu'elle donne, semble à Oldham être un Gordius ou un Mermis. Il s'agit certainement d'un Mermithidé, vu que les Gordius sont rarissimes chez les Chrysomélides.

Bovien (1932) mentionne également des Nématodes chez les Phyllotreta sans donner de précisions. Il s'agit vraisemblablement d'Anguillulidæ.

\section{Conclusion et Résumé}

L'on remarque par ce qui précède que seulement 16 Chrysomélides sont signalés comme hébergeant des Helminthes et que, si l'on considère le nombre d'espèces connues de cette famille de Coléoptères (plus de 25.000), il doit y avoir d'innombrables autres hôtes et parasites non étudiés jusqu'ici.

Ceci est un exemple frappant de nos lacunes en ce qui concerne les parasites de Coléoptères.

Parmi les Némathelminthes, les Mermithidés de Chrysomélides semblent appartenir essentiellement au genre Hexamermis, et peutêtre à l'espèce $H$. albicans $\mathrm{V}$. Sieb.

Les Anguillulides (Howardula) ne se rencontrent que chez les Galerucidæ et Halticidæ.

Enfin, un seul Gordiacé : Parachordodes violaceus (Baird), est mentionné dans la littérature.

D'autre part, les expériences de Denton montrent que des Chrysomélides peuvent servir de second hôte intermédiaire de certains Trématodes, dont le premier est un Mollusque et le définitif un Oiseau.

Le tableau qui suit résume de façon synoptique nos connaissances sur les Helminthes de Chrysomélides.

\section{Bibliographie}

Aвrahams (R.). - Mermithiden als Parasiten das Kartoffelkäfers (Leptinotarsa decemlineata Say). Zeitschr. f. Pflanzenkrank. u. Pfanzenschutz., XI, 1938, 507-513.

Bovien (P.). - On a new Nematode Scatonema wïel:eri gen. et sp. nov. parasitic in the body cavity of Scatopse fuscipes Meig. (Dipt. Nematocera). Vid. Med. Daresk. Nat. For., XCIV, 1932, 13-32, 7 fig.

Chitrenden (F. H.) et Marsh (H. O.). - The Western cabbage flea beetle. U.S. Dept. Agric. Bull., 1920, 902, 1-21.

Coвв (N. A.). - Howardula benigna, a new parasite of the cucumber beetle. Science $n$ s., LIX, 1921, $\mathrm{n}^{\circ} 1409,667-670$. 


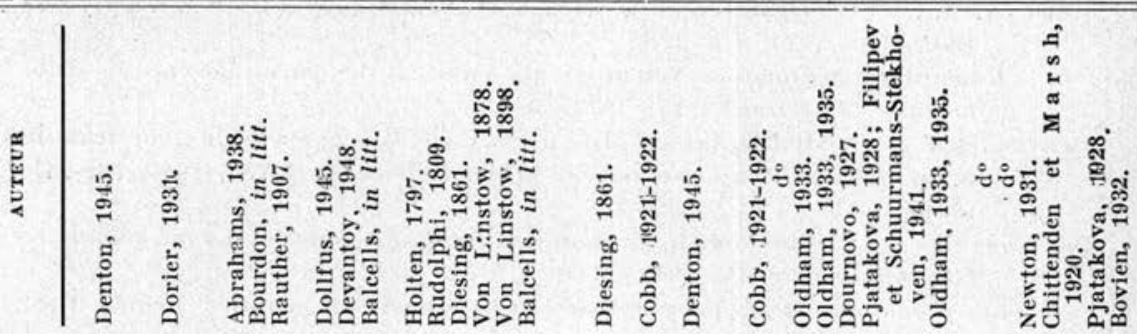

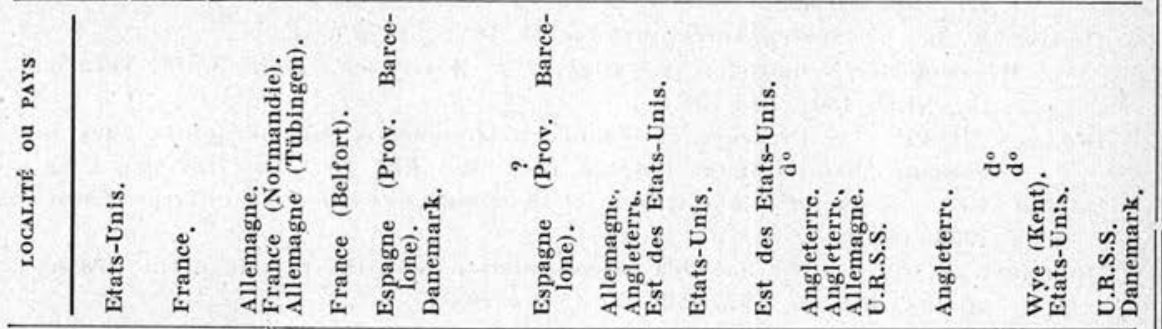

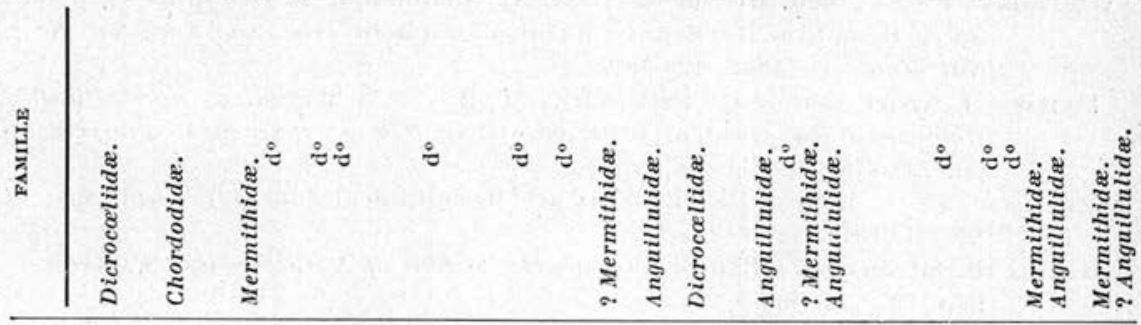

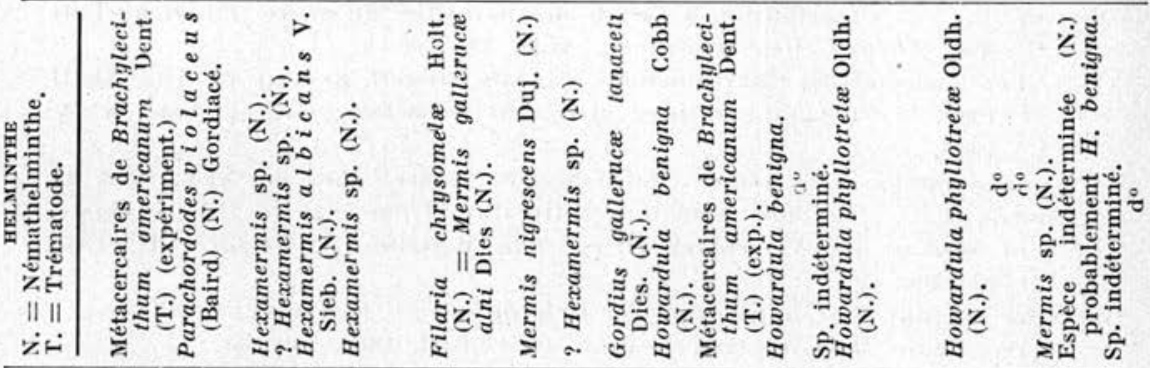

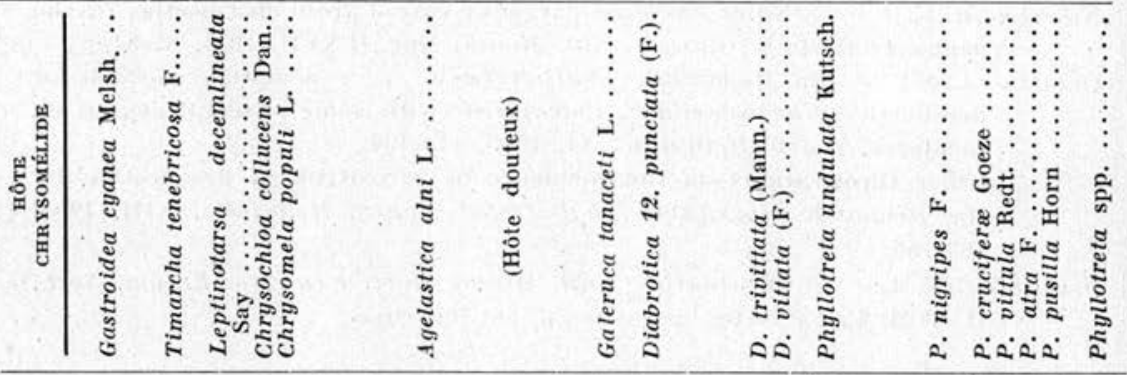


Совв (N. A.). - Contribution to a science of Nematology VII. Waverley Press Baltimore, 1922, 1-4, 4 fig.

Howardula benigna, a Nematode parasite of the cucumber beetle (Diabrotica). J. Parasit., IX, 1922, 36.

Denton (J.-F.). - Studies on the life-history of Brachylecithum americanum n. sp., a liver fluke of passerin birds. J. Parasit., XXXI, 1945, 131-141, 10 fig.

Devantox (J.). - La Chrysomèle du peuplier Melasoma populi ou Lina populi L. L'Entomologiste, IV, 1948, 74-81.

- Les prédateurs et les parasites de la Chrysomèle du peuplier. Feuille Nat., III, $1948,85-89$.

DIEsIng (K. M.). - Systema helminthum, II, 1851, 588 p.

- Revision der Nematoden. Sitzungb. d. k. Wissensch., Wein. Math. Naturw. Cl., XLII, 1861, 595-736.

Dollfus (R.-Ph.). - Présence en France d'Allomermis myrmecophila chez la Fourmi Lasius alienus Forster. Bull. Soc. Ent. Fr., 1945, 102-105, 2 fig.

DORIER (A.). - Recherches biologiques et systématiques sur les Gordiacés. Thèse, 1930, 183 p.

Dounnovo (Z. E.). - The flax-flea in conjunction with the period of the sowing of flax. Moscou, 1927, 301, 21-43 (en russe).

Dujardin (F.). - Mémoire sur la structure anatomique des Gordius et d'un autre Helminthe, le Mermis, qu'on a confondu avec eux. Ann. Soc. Sc. Nat. Zool., II, 1842, 129-151.

Filipjev (I. N.) et Schucrmans-Steknoven (J. H.). - A Manual of Agricultural Ilelmintroiogy, Leiden, Brill. éd., 1941,878 p. (rarasites d'insectes, pp. $725-878)$.

Hagmeier (A.). - Beitrag zur Kienntnis der Mermithiden. Züol. Jchr. Abt. Syst., XXXII, 1912, 521-612, 5 pl.

Holten (H. S. von), - « Filaria Chrysomelie ». Skr. af Naturh-Selsk., Kiobenh., IV, 1797, 16-18.

Joliver (P.). - Contribution à l'étude des parasites du genre Timarcha Latr. (Col. Chrys.), Miscell. Entom., XLII, 1945, 8-11.

- Les Parasites des Chrysomélides français. Miscell. Entom., $1^{\text {ro }}$ liste, XLIII, 1946, 87-92 ; ibid., 2e liste, XLIV, 1947, 108-109 ; ibid., $3^{e}$ liste, XLIV, 1948, 52-54.

- Introduction à la biologie des Timarcha. Miscell. Entom., XLV, 1948, 25.

LA Rivens (I.). - Entomic Nematode Litterature from 1926 to 1946, exclusive

of Medical and Veterinary Titles. The Wasmann Collector, VII, 1949, 177-206.

Linstow (O. von). - Compendium der Helminthologie, Hannover, 1878, 382 p.

Das Genus Mermis. Arch. f. Mikr. Anat., LIII, 1888, 149-168.

Newton (H. C. F.). - Notes on some parasites reared from flea-beetles of the genus Phyllotreta (Chrys.). Ent. Month. Mag., LXVII, 1931, 82-84.

OLdham (J. N.). - On Howardula phyllotreta n. sp. a nematode parasite of flea-beetles (Chrysomelida : Coleoptera) with some observations on its incidence. Journ. Helminth., XI, 1933, 119-136.

- Further Observations on the incidence of parasitism of flea-beetles (by the Nematode Howardula phyllotretæe). Journ. Helminth., XIII, 1935, $163-166$.

Pjatakova (V. L.). - Flea-beetles. Bull. Mleew. Hort. Exp. Sta. Entom. Sect., II, 1928, 75 p., 70 fig. (parasites p. 56) (en russe). 
Rauther (M.). - Beiträge zu Kenntnis von Mermis albicans V. Sieb. mit besonderer Berücksichtigung des Haut-Nerven-Muskelsystems. Zool. Jahrb. (Anat.), XXIII, 1907, 1-76.

RUDOLPHI (C. A.). - Entozoorum sive vermium intestinalinm historia naturalis, II, 1809,457 p.

Zwaluwenburg (R. H. van). - The Interrelationships of Insects and Roundworms. Bull. Exp. Station Hawaiian Sugar Planters'Assoc. Ent. Ser. Bull., $\mathrm{n}^{\circ} 20,1928,5-68$.

Institut Royal des sciences naturelles de Belgique (Bruxelles) et Laboratoire Arago, Banyuls-sur-Mer (P.-.O.) 